\title{
Die Unterschiede in den Reactionen von Strychnin und Gel-
} semin hat $\mathrm{Raimond} \mathbf{i}^{*}$ ) zusammengestellt, da die beiden Alkaloide in vielfacher Hinsicht solche Aehnlichkeit zeigen, ${ }^{* *}$ ) dass eine Verwechslung leicht möglich ist.

Die zur Vermeidung eines Irrthums zu beachtenden Verschiedenheiten sind: Bei Berührung mit Kaliumbichromat und Schwefelsäureterhydrat gibt Gelsemin eine himmelblaue Farbe, mit grünen Streifen durchsetzt, die endlich ganz in Grün übergeht, während Strychnin bei gleicher Behandlung eine violette, durch Rothblau in Ziegelroth übergehende Färbung gibt, welche beim Verdünnen gelb wird. Concentrirte Schwefelsäure löst Gelsemin mit gelbbrauner, beim Erwärmen purpurroth werdender Farbe, das Strychnin dagegen in der Kälte farblos, beim Erwärmen mit gelbbrauner Farbe. Concentrirte Schwefelsäure und Zucker färben das Gelsemin rothblau, das Strychnin in der Kälte nicht. Ueberchlorsäure wird mit Gelsemin gelb, mit Strychnin lebhaft roth.

Ferridcyankalium enthaltendes Eisenchlorid gibt mit Gelsemin, aber nicht mit Strychnin, eine grüne Färbung. Strychnin in Schwefelsäurebihydrat gelöst und mit etwas Chlorkalium versetzt, gibt beim Erwärmen eine rothbraune Färbung, die bei gleicher Behandlung des Gelsemins nicht auftritt.

Ueber das Dehydromorphin (Oxydimorphin, Pseudomorphin), das bei der Einwirkung verschiedener Oxydationsmittel auf Morphin aus letzterem entsteht, liegen neuere Mittheilungen von Jul. Donath***) o. Hesse $\dagger$ ) and Karl Polstorff $\mathrm{K}^{\prime}$ ) vor, aus denen hervorgeht, dass die Zusammensetzung $\mathrm{C}_{17} \mathrm{H}_{18} \mathrm{NO}_{3}$ ist. Bezüglich der Beweise hierfür sei auf die Originalabhandlungen hingewiesen. Da das Pseudomorphin viele Reactionen mit dem Morphin gemeinsam hat, z. B. die mit Eisenchlorid, mit concentrirter Salpetersäure, mit Fröhde 'schem Reagens und mit Jodsäure, so gibt Hesse folgende Unterscheidungsreactionen an.

Morphin löst sich in reiner, concentrirter Schwefelsäure mit sehr schwach röthlicher Farbe, (keineswegs farblos, wie vielfach angegeben

*) Ann. chim. med. farm. 1885, pag. 166; durch Archiv der Pharmacie [3 R.] 24, 139.

**) Vergl. z. B. diese Zeitschrift 22, 482.

***) Journal für praktische Chemie [N. F.] 33, 559; vom Verfasser eingesandt.

†) Liebig's Annalen der Chemie 234, 253 und 235, 229.

††) Ber. d. deatsch. chem. Gesellsch. z. Berlin 19, 1760. 
wird), Pseudomorphin anfangs farblos, doch wird die Lösung bald gelblich und dann röthlich. Enthält die Säure ganz wenig Eisenoxyd*), so wird mit Morphin eine röthlich gefärbte, mit Pseudomorphin eine schön blau gefärbte Lösung erzielt. Allein letztere Lösung wird bald intensip violett und schliesslich braungrün.

Wird Morphin mit dem gleichen Gewicht Rohrzucker innig vermengt, so löst es sich nun in reiner oder eisenoxydhaltiger, concentrirter Schwefelsäure violettroth. Unter den gleichen Verhältnissen gibt Pseudomorphin mit reiner Schwefelsäure eine intensiv dunkelgrün gefärbte, allmählich braungrün werdende Lösung. In eisenoxydhaltiger Säure löst sich dagegen das Gemenge von Pseudomorphin und Rohrzucker prächtig blau, später intensiv dunkelgrün werdend.

Wendet man anstatt Rohrzucker, Milchzucker oder Glykose an, so löst sich das Pseudomorphingemenge in reiner, concentrirter Schwefelsäure anfangs farblos, nach etwa einer Stunde wird diese Lösung grünlich and nach mehreren Stunden prächtig blaugrün. Bei Anwendung von eisenoxydhaltiger Säure treten auch hier die Farbenreactionen rascher und intensiver ein.

Dieselben Reactionen wie das Pseudomorphin gibt auch das Diacetylpsendomorphin.

Donath gibt folgende unterscheidende Reaction an ${ }^{* *}$ ): Man übergiesst in einem Porzellanschälchen etwas Dehydromorphin mit etwa 8 Tropfen einer Schwefelsäure, welche auf 2 Volumina concentrirter Säure 1 Volumen Wasser enthält, und erwärmt, vorsichtig schwenkend, auf einem Flämmchen, bis Schwefelsäuredämpfe sich zu entwickeln beginnen, wobei die ganze Flüssigkeit schön blaugrün wird. Verdünnt man vorsichtig mit Wasser, so wird sie rosenroth und wenn man nun Oxydationsmittel zusetzt (1-2 Tropfen concentrirte Salpetersänre, oder 1 Tropfen einer Lösung von 1 Theil Natriumnitrit in 20 Theilen Wasser, oder endlich 1 Tropfen einer Lösung von unterchlorigsaurem Natron) so wird die Lösung prachtvoll tiefviolett gefärbt. Morphin wird bei gleicher Behandlung durch das Erhitzen mit reiner Schwefelsäure rosenroth und bald missfarbig bräunlich,

*) Eine solche Säure wird erhalten, wenn man zur concentrirten Säure ganz wenig Eisenehlorid hinzubringt.

**) Dieselbe ist in etwas anderer Form von Husemann für Morphin vorgeschlagen worden (vergl, diese Zeitschrift $\mathbf{3}, 149$ ). 
beim Verdünnen mit Wasser röthlich and durch die genannten Oxydationsmittel prächtig himbeerroth.

In einer besonderen Abhandlung gibt J. Donath*) sodamn noch zwei Morphinreactionen an, die auch zu einer Unterscheidung von Dehydromorphin (Pseudomorphin) dienen können.

1) Die zuerst von Tattersall**), dann von Vitali***) angegebene Reaction des Erhitzens mit concentrirter Schwefelsäure und arsensaurem Natron hat der Verfasser dadurch erweitert, dass er die mit Wasser verdünnte Masse mit Chloroform ausschüttelt. Donath beschreibt die Reaction etwas abweichend von den genannten Autoren folgendermaassen.

Fein zerriebenes Morphin (etwa $1 \mathrm{mg}$ ) wird im Porzellanschälchen mit 8 Tropfen concentrirter Schwefelsäure innig verrührt, ein kleines Körnchen Kaliumarseniat zugefügt und damit verrieben. Erhitzt man unter Umschwenken auf einem Flämmchen bis zum beginnenden Entweichen von Säuredämpfen, dann entsteht eine schöne, blauviolette Färbung, die bei weiterem Erwärmen dunkel braunroth wird. Beim vorsichtigen Veräünnen mit Wasser entsteht eine röthliche Färbung, welche bei weiterem Wasserzusatz grün wird. Giesst man diese Flüsigkeit in eine Eprouvette, fügt Chloroform hinzu and schüttelt, dann färbt sich letzteres prächtig violett. Ebenso färbt sich Aether schön violettroth, während die darunter befindliche Flüssigkeit braun ist.

Dehydromorphin dagegen wird beim Verreiben mit Schwefelsäure und Kaliumarseniat schmutzig grün, beim Erwärmen braun und nach dem Verdünnen mit Wasser intensiv grün. Doch gibt diese Lösung an Chloroform keinen Farbstoff ab.

Hinsichtlich dieser Reaction bemerken Gasselin und Lévy $\dagger$ ), dass sie auch obne einen Zusatz von arsensaurem Natron gelinge, wie dies auch Jorissen $\dagger$ ) früher gezeigt habe, der allerdings eine Behandlung der wässerigen Lösung mit Chloroform ebenfalls bereits angibt.

Die zweite Reaction, welche Donath mittheilt, beruht auf der

*) Journal für praktische Chemie [N.F.] 33, 563; vom Verfasser eingesandt.

**) Vergl. diese Zeitschrift $\mathbf{2 0}, 119$.

***) Vergl. diese Zeitschrift 21, 5\$1.

†) Journ. de Pharm. et de Chim. [5. Sér.] 14, 458; durch Chemikerzeitung 10, (Repertorium) 256.

††) Vergl. diese Zeitschrift 20, 422. 
Einwirknng von Schwefelsäure und chlorsaurem Kali auf das Morphin und ist der Eisenchloridreaction ähnlich.

Etwas Morphin mit ungefähr 8 Tropfen concentrirter Schwefelsäure verrieben, wird anf Zusatz eines Tropfens einer Lösung von 1 Theil Kaliumchlorat auf 50 Theile concentrirter Schwefelsäure in der Kälte schön grasgrün, welche Farbe sich lange hält. Am Rande der Flüssigkeit zeigt sich eine schwach rosenrothe Färbung.

Dehydromorphin wird unter denselben Umständen braungrün.

Dieselben Reagentien hat, wie auch Donath anführt, schon früher Vitali*) in etwas anderer Weise zum Hervorrufen einer Morphinfarbenreaction vorgeschlagen.

Zum Nachweis des Traubenzuckers empfiehlt C. Agostini**) fünf́ Tropfen der zu untersuchenden Flüssigkeit mit fünf Tropfen einer Goldchloridlösung $(1: 1000)$ und zwei Tropfen Kalihydratlösung $(1: 20)$ zum Sieden zu erhitzen. Nach dem Abkühlen tritt, wenn Traubenzucker zugegen war, eine je nach der Menge desselben mehr oder weniger intensive, prächtig violette Färbung auf. Die Reaction gestattet den Zucker noch in einer Verdünnung von 1/10000 nachzoweisen und eignet sich namentlich auch zur Untersuchung von Urin auf Zucker, da der Verfasser durch die Untersuchung von etwa 100 normalen und pathologischen Harnen gefunden hat, dass ausser Eiweiss keiner der normalen und anormalen Bestandtheile des Urins die Färbung hervorbringt.

Untersuchungen über die Darstellung und die Eigenschaften des Inosits, sowie dessen Verbreitung im Pflanzenreiche hat Richard Fick***) angestellt und Maquenne $\dagger$ ) hat Studien über die Darstellung, Eigenschaften und Constitution des Inosits, sowie über die Indentität dieses Körpers mit Dambos e veröffentlicht.

Ich muss mich darauf beschränken, auf diese ausführlichen Abhandlungen hinzuweisen.

*) Vergl. diese Zeitschrift 21, 581. Daselbst ist, um jedes Missverständ. niss auszuschliessen, in dem vorletzten Satze "Tropft man etc." hinter den Worten "eine Lösung von chlorsaurem Kali" einzuschaiten: "in concentrirter Schwefelsäure".

**) Ann. di Chim. Farm.; Journ. Pharm. Chim. [5.] 14, 464; durch Chem. Centralblatt [3. F.] 18, 99.

***) Pharm. Zeitschrift f. Russland 26, 81 .

†) Bull. de la société chim. de Paris 47, 290. Comptes rendus 104, 225, 297 und 1853. 